\title{
Ultrastructural and Antioxidant Studies of Etoposide Treated Kidney of Rat
}

\section{Pratibha Kamble ${ }^{1 *}$, Sameer Kulkarni ${ }^{2}$ and Dayanand A. Bhiwgade ${ }^{3}$}

${ }^{1}$ Department of Life Sciences, University of Mumbai, Kalina, Santacruz (E) 400098, Mumbai, India ${ }^{2}$ Department of Biochemistry, Grant Medical College and Sir J.J.Group of Hospitals, Byculla, Mumbai, India

${ }^{3}$ Department of Biological Sciences, D.Y.Patil University, Belapur, Navi Mumbai, India

\begin{abstract}
Etoposide is well-known drug long been used in clinical trials for treating various cancers. The beneficial action of drug can be used in treatment of small cell and non-small cell lung cancer, lymphoma and ovarian cancer.

In this study we examined the effect of etoposide on morphological structure of renal tissue. Studies showed that long-term treatment of etoposide given at dose of $1 \mathrm{mg} / \mathrm{kg}$ i.p for period of 8 weeks resulted in histological changes that are associated with dilated proximal convoluted tubules (PCT) with enlarged lumen (L) and vacuolation in their epithelium around nucleus. The onset of necrosis and atrophied glomerulus is also observed. Additionally, ultrastructural studies showed presence of mitochondria in segments and lumen with broken microvilli.

Furthermore, biochemical finding's in etoposide treated kidney displayed significant increase in Glutathione-STransferase (GST), Glutathione Peroxidase (Gpx) and decrease in glutathione reductase (GR), gamma glutamyl transpeptidase (GGT) activities however, glutathione (GSH), Catalase (CAT) and Lipid peroxidation (MDA Content) showed non-significant decrease.

The drug metabolizing enzyme's Cytochrome p450 (Cyp450) showed no change whereas, Cytochrome b5 (Cyp b5) showed significant decrease. Thus we conclude that etoposide does not cause nephrotoxic effect at given dose level.
\end{abstract}

Keywords: Etoposide; Kidney; Glomerulus; Mitochondrial; Glutathione; Free radicals

\section{Introduction}

Podophyllotoxins has been recognised as active compound used potentially for treatment of various cancers. The compound can be used for further research due to its biomolecular mechanisms that targets cancer cell proliferation.

Some of the hemisynthetic compounds such as etoposide and teniposide and number of new drugs that are now emerging are podophyllotoxins that are currently used as a cancer chemotheraupatic agent [1]. Perhaphs its wide spread application in clinical oncology is often been mitigated due to its toxicity. The beneficial use of podophyllotoxins is often restricted due to its toxic effects, as the drug might cause serious gastrointestinal irritation.

Etoposide, is cell-cycle phase-specific drug that delays and kills the cell in the late S or G2 phase. It's useful activity has been established in the therapy of small cell and non-small cell lung cancer, lymphoma, ovarian cancer and kaposi's sarcoma [2].

Previous studies have shown various actions of etoposide reporting that the drug can oxidize GSH and protein $\mathrm{SH}$ groups in HL-60 cells, also etoposide treatment cannot amplify the loss of essential antioxidants, but increases lipid hydroperoxide concentration in serum [3-5].

However, studies on etoposide induced renal toxicity has been least documented. Current investigations were therefore carried out with an aim to determine the effect of long-term treatment of etoposide drug regimens on histopathological, ultrastructural and antioxidant status in kidney of rat. Additionally the role of drug metabolizing enzymes Cytochrome p450 and b5 in etoposide activation is also studied.

\section{Materials and Methods}

\section{Animals and ethical clearance}

Adult male albino rats of Wistar strain were used for the study. Animals were weighing about 220-250g obtained from Rajudyog biotechnology division Maharashtra, India were used. The animal studies were carried out upon institutional animal ethical committee approval.

\section{Drugs}

Etoposide was procured from Dabur India Ltd. Bovine serum albumin, reduced and oxidized GSH, thio barbituaric acid (TBA), butylated hydroxytolune (BHT), L-gammaglutamyl-p-nitroanilidehydrochloride, sodium azide, Dinitro thio benzoic acid (DNTB), $\mathrm{NADPH}$, sodium dithionite, sodium formate were purchased from SRL India. All other chemicals used were of analytical grade.

\section{Methodology}

Animals were acclimatized for a period of two-weeks and were then treated. They received standard pellet and water ad libitum. Rats

*Corresponding author: : Pratibha R. Kamble, Department of Life Sciences, University of Mumbai, Kalina, Santacruz (E) 400098, Mumbai, India, Tel: 6143307068 ; E-mail: kamblepratibha@gmail.com

Received February 23, 2013; Accepted March 11, 2013; Published March 13 2013

Citation: Kamble P, Kulkarni S, Bhiwgade DA (2013) Ultrastructural and Antioxidant Studies of Etoposide Treated Kidney of Rat. J Cancer Sci Ther 5: 137 141. doi:10.4172/1948-5956.1000199

Copyright: $\odot 2013$ Kamble $\mathrm{P}$, et al. This is an open-access article distributed under the terms of the Creative Commons Attribution License, which permits unrestricted use, distribution, and reproduction in any medium, provided the original author and source are credited. 
were coded in groups of two per cage and then were subsequently examined for further study. Experimental rats were injected with 1.0 $\mathrm{mg}$ of etoposide per kg i.p daily for a period of 8 weeks. Control group received $0.5 \mathrm{ml}$ of saline daily along with the treated set of the rats. The change in the body weight was monitored per week.

At termination, rats were sacrificed using ether anaesthesia. The kidneys were dissected out, washed in ice-cold saline, blotted and a homogenate was prepared in $0.1 \mathrm{M}$ sodium phosphate buffer $(\mathrm{pH} 8.0)$. Homogenate was further centrifuged and the supernatant fractions obtained were utilized for the analysis of GSH- related enzymes like GR, Gpx, GGT, GST and CAT [6-10]. Trichloroacetic acid (TCA) treated samples were utilized for the estimation of reduced GSH and Lpx $[11,12]$. The resulting supernatant fractions were recentrifuged for an additional $60 \mathrm{~min}$ at $105,000 \mathrm{~g}$. The microsomal pellets obtained were carefully collected and used for the estimation of CYP450 and Cytochrome b5 [13]. Total protein content was estimated by Lowry et al. [14]. All spectrophotometric readings were taken on Shimadzu UV160 double beam spectrophotometer.

\section{Analysis}

The significance of difference between the means was calculated by students t-test and results were expressed as mean \pm SEM. Significance between both groups controls and etoposide is shown as ${ }^{*} \mathrm{p}<0.05$.

\section{Histopathological studies}

Light microscopy: Light microscopy was performed to study the histological changes of kidney by using paraffin method as described earlier [15]. Kidney tissue was cut into pieces of desired size and fixed into Bouin's fixative (standard aqueous picric acid $75 \mathrm{ml}+$ conc. Formalin $25 \mathrm{ml}+$ glacial acetic acid $5 \mathrm{ml}$ ) for $24-48 \mathrm{~h}$. Tissues were removed from fixative solution and washed thoroughly with distilled water for few hours so as to remove extra Bouin's fixative.

After fixation, samples were washed with $70 \%$ alcohol to remove excess of picric acid from the tissues and dehydrated in graded series of ethanol. Tissues were dealcoholized and clear tissues were kept in filtered molten paraffin which was then sectioned to $3.0 \mu \mathrm{m}$ thick series of section using rotary microtome (Microme, Model No. HM 310). The sections were stained with hematoxylin followed by eosin as secondary stain. The slides were mounted by using DPX mountant as an adhesive agent. The (x20) magnification was used to observation.

Transmission electron microscopy: Kidney tissue was removed from the animals instantaneously to their been anesthesized and killed by decapitation, sliced into one $\mathrm{mm}$ pieces in a drop of $3 \%$ glutareldehyde. Tissue was then immersed in fresh ice cold fixative for two hours and then in $0.1 \mathrm{M}$ cacodylate buffer for next $4 \mathrm{~h}$. The tissues was then rinsed briefly in buffer and post osmicated in $1 \%$ osmic acid for one to two hours.

Kidney was dehydrated in an ascending series of alcohol, followed by propylene oxide and finally embedded in resin that was polymerized at $600^{\circ} \mathrm{C}$. Subsequently the blocks were prepared in araldite and $1 \mathrm{~m}$ sections were cut with a glass knife on LKB-2000S, ultramicrotome mounted on glass slides and stained with buffered toluidine blue. Appropriate areas were selected with the light microscope. Finally, ultrathin sections of selected blocks were cut with a diamond knife, picked up on copper grids and stained with uranil acetate and lead citrate for final viewing. The ultrathin sections were scanned and photographed on JEM-JEOL 100s electron microscope. Maginification used to observe the sectioned tissue were $(\mathrm{x} 10,000)$.

\section{Results}

\section{General health}

Etoposide treated rats showed zero mortality rate during entire duration of treatment. One of significant effect of etoposide treated rat observed was steady increase in weight, coupled with sluggishness and fluppyness. Drug did not cause any irritation at the site of the injections.

\section{Body weight and organ weight}

Total bodyweight of 8 weeks control and etoposide treated animals are shown in Table 1. Control rats registered a uniform increase in body weight throughout the entire duration of treatment. Etoposide treated rat showed significant difference when compared to controls.

Animals injected with etoposide showed increase in fat deposition which was noticed at the time of dissection this could be one of the side effects caused by the drug.

Kidney of etoposide treated group of rat's revealed non-significant decrease in weight compared to controls (Table 1).

Table 1 depicts total protein content and Lpx values of kidney. The mean values were expressed as $\mathrm{mg} / \mathrm{gm}$ tissue. Total protein content registered significant increase of $325.27 \pm 19.11$ in etoposide treated rats as compared to control rats reported as $262.73 \pm 17.35$ (Table 1). Lpx (MDA content) formed after etoposide treatment showed nonsignificant decrease $0.258 \pm 0.015$ compared to control rats shown as $0.31 \pm 0.03$.

The mean values of GSH in etoposide treated group showed nonsignificant decrease $7.724 \pm 0.732$ compared to control rats showed 8.06 \pm 0.74 . Gpx showed significant increase of $565.76 \pm 29.5$ as compared to control rats shown as $415.4 \pm 25.14$. GGT activity of etoposide treated

\begin{tabular}{|l|c|c|}
\hline \multirow{2}{*}{ Parameter } & \multicolumn{2}{|c|}{ Groups } \\
\cline { 2 - 3 } Body weight $(\mathrm{g})$ & Control $(\mathbf{n = 5})$ & Etoposide (n=5) \\
\hline Organ weight (g) & $367 \pm 12.31$ & $304 \pm 14.352^{*}$ \\
\hline Protein (mg/g tissue) & $1.261 \pm 0.045$ & $1.168 \pm 0.0269 \#$ \\
\hline $\begin{array}{l}\text { Lipid peroxidation (nmol of Malondialdehyde } \\
\text { formed per mg protein) }\end{array}$ & $262.73 \pm 17.35$ & $325.27 \pm 19.11^{*}$ \\
\hline
\end{tabular}

Etoposide rats versus control rats: * $p<0.05$, \# non- significant

Table 1: General and stress-induced parameter in Etoposide-treated and contro rats (mean \pm SEM).

\begin{tabular}{|l|c|c|}
\hline \multirow{2}{*}{ Parameter } & \multicolumn{2}{|c|}{ Groups } \\
\cline { 2 - 3 } Glutathione $(\mu \mathrm{mol} / \mathrm{mg}$ protein) & Control $(\mathbf{n = 5})$ & Etoposide $(\mathbf{n}=5)$ \\
\hline $\begin{array}{l}\text { Glutathione-s-transferase (nmol of } \\
\text { cDinitrobenzene bound/mg protein) }\end{array}$ & $1.46 \pm 0.11$ & $6.355 \pm 0.35$ * \\
\hline Glutathione reductase (nmoles/mg protein) & $9.98 \pm 0.37$ & $8.304 \pm 0.611^{*}$ \\
\hline $\begin{array}{l}\text { Glutathione peroxidase ( } \mu \text { gm utilized/min/ } \\
\text { mg protein) }\end{array}$ & $415.4 \pm 25.14$ & $565.76 \pm 29.5^{*}$ \\
\hline $\begin{array}{l}\text { Gammaglutamyltransferase }(\mu \mathrm{mol} \text { of } \\
\text { p-nitroaniline/ min/mgprotein) }\end{array}$ & $9.092 \pm 0.02$ & $7.749 \pm 0.118^{*}$ \\
\hline Catalase (unit/mg protein) & $0.617 \pm 0.09$ & $0.533 \pm 0.052 \#$ \\
\hline Cytochrome p450 (nmol/mg protein) & $683.4 \pm 40.5$ & $681.18 \pm 66.95 \#$ \\
\hline Cytochrome b5 (nmol/mg protein) & $2605.5 \pm 259.2$ & $555.81 \pm 49.6^{*}$ \\
\hline
\end{tabular}

Etoposide rats versus control rats: ${ }^{*} p<0.05$, \# non-significant

Table 2: Glutathione and antioxidant enzyme activity of Kidney from Etoposidetreated and control rats (mean \pm SEM) 
group revealed significant decrease shown as $7.749 \pm 0.118$ as compared to the controls shown as $9.092 \pm 0.02$ (Table 2).

After etoposide treatment the activity of GST was significantly higher as compared to control group. The mean values shown as 6.355 \pm 0.35 in etoposide treated rats as compared to controls as $1.46 \pm 0.11$ (Table 2). The mean values of GR activity reported significant decrease as $8.304 \pm 0.611$ as compared to controls shown as $9.98 \pm 0.37$ (Table 2).

A non-significant decrease in mean values $0.533 \pm 0.052$ is noticed after etoposide treatment as compared to controls $0.617 \pm 0.09$. CAT showed non-significant decrease of $0.533 \pm 0.052$ compared to controls shown as $0.617 \pm 0.09$ (Table 2).

The level of drug metabolizing enzyme namely CYP450 in kidney showed non-significant change in values reported as $681.18 \pm 66.95$ and controls showed $683.4 \pm 40.5$ whereas, Cytochrome b5 level showed significant decrease shown as $555.81 \pm 49.6$ after drug treatment as compared to controls seen as $2605.5 \pm 259.2$ (Table 2).

\section{Light microscopy of kidney}

Tissue section of the isolated kidney from etoposide treated rats exhibited marked congestion of the glomeruli with glomerular atrophy (Figures 2 and 3). The proximal convoluted tubule (PCT) shows vacuolation in its epithelial lining and enlarged lumen (Figures 1-3), also presence of prominent nucleolus (NU) in almost all the nuclei are seen (Figure 1). Desquamation in particular was not noticed, but damage to the brush borders of the cell and prescence of debries in tubular lumen was apparent (Figures 1 and 3).

\section{Electron microscopy of kidney}

Electron Micrographs reveal several ultrastructural changes in S1, S2 and S3 segments of the proximal tubules (Figures 4-6). The S2 segment showed presence of scanty microvilli of variable height (Figure 5) but S3 segment showed complete abscence of microvilli (Figure 6). Presences of many mitochondria of variable lengths with dense matrix were visible among the basolateral foldings (Figures 4 and 5). Condensation of nuclear chromatin and appearance of lysosomal bodies (Figure 7) were seen. SER with amorphous material was also visible (Figure 7). The presence of necrotic debris (Figure 6) in the tubular lumen with tubular damage was an indication of onset of necrosis.

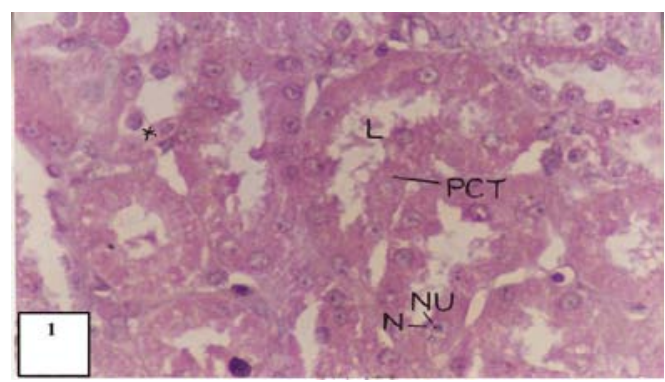

Figure 1: Light Micrographs of rat kidney treated with Etoposide for 8 weeks. Light micrographs of rat kidney treated with etoposide for 8 weeks shows the presence of dilated proximal convoluted tubules (PCT) with enlarged lumen $(\mathrm{L})$ and vacuolation $\left({ }^{*}\right)$ in their epithelium around the nucleus $(\mathrm{N})$. The prominent nucleolus (NU) in almost all the nuclei are also seen. Magnification taken (x 40).

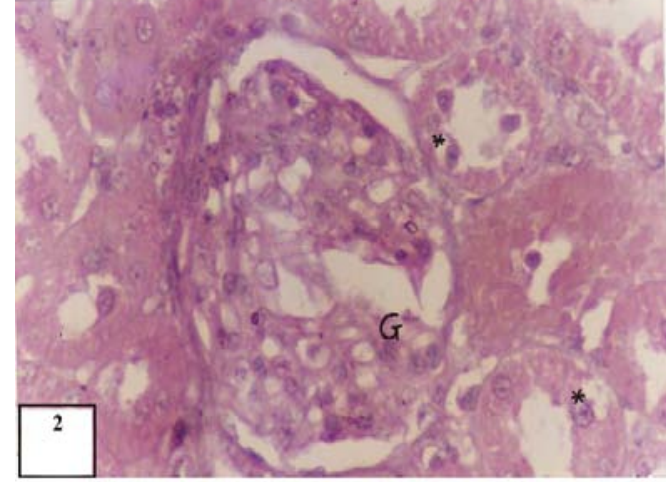

Figure 2: Light Micrographs of rat kidney treated with Etoposide for 8 weeks. Note the onset of necrosis and atrophied glomerulus $(G)$. Large vacuoles $\left(^{*}\right)$ in the epithelium of tubules. Acute loss of brush border of the tubular lumen (thin arrow). Magnification taken (x 40).

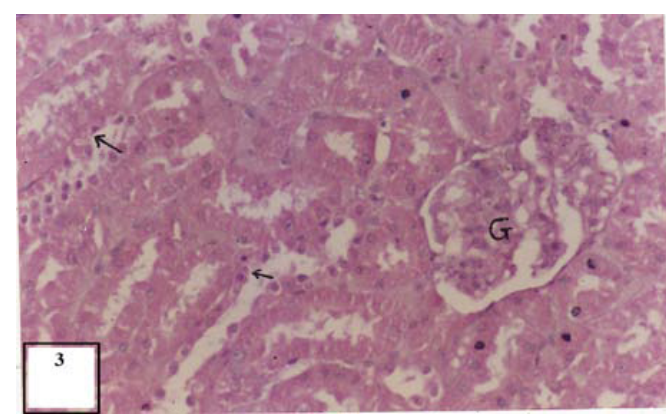

Figure 3: Light Micrographs of rat kidney treated with Etoposide for 8 weeks. Note the onset of necrosis and atrophied glomerulus (G). Large vacuoles $\left(^{*}\right)$ in the epithelium of tubules. Acute loss of brush border of the tubular lumen (thin arrow). Magnification taken (x 40).

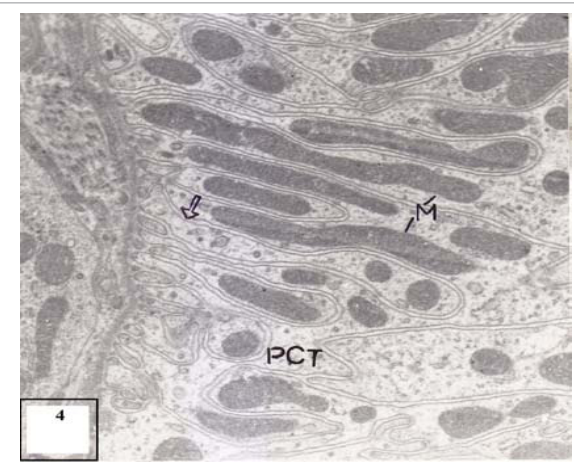

Figure 4: Electron Micrographs of rat kidney treated with Etoposide for 8 weeks. Electron Micrographs of S1 segment of proximal convoluted tubule (PCT) shows large number of dense mitochondria (M) among the basolateral folding of the tubule (hollow arrow). Magnification taken (x 15,000).

\section{Discussion}

A present study documents the mechanism of the selective action of etoposide and its role in causing nephrotoxicity. Till now studies on long-term treatment of etoposide and its nephrotoxic effect has been least documented in clinical oncology. The primary aim of this study was to gain insight into the possible mechanism of the toxic effects of the drug on renal tissue.

Histopathological studies of kidney showed that long-term treatment with etoposide induces structural alterations, which is 


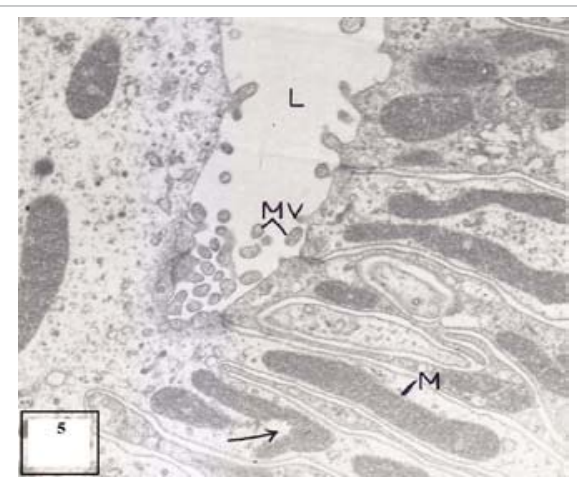

Figure 5: Electron Micrographs of rat kidney treated with Etoposide for 8 weeks. Electron Micrographs of S2 segment shows dense mitochondria (M) with occasional ruptures (thin arrow) and lumen (L) with broken microvilli (Mv). Magnification taken (x 15,000).

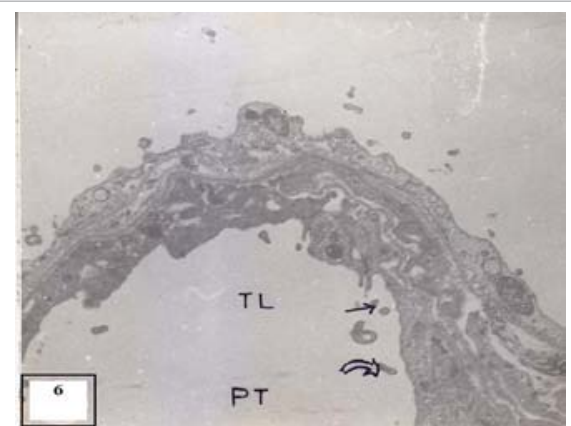

Figure 6: Electron Micrographs of rat kidney treated with Etoposide for 8 weeks. Electron Micrographs showing the proximal tubules (PT). The noticeable features are loss of microvilli (Mv) (thin arrows). Also note the tubular lumen ( $T L$ ) filled with necrotic debris (curled arrow). Magnification taken $(x 20,000)$

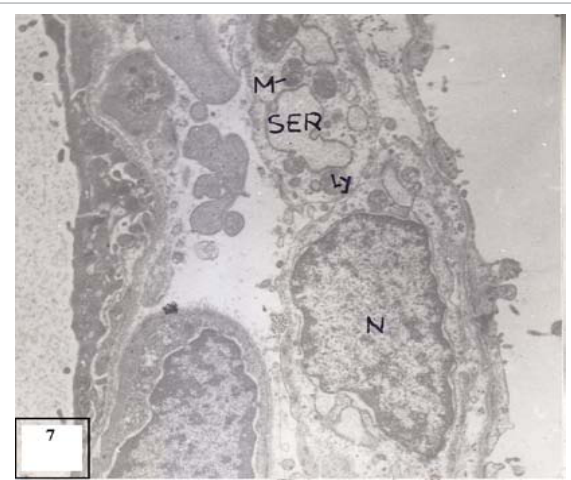

Figure 7: Electron Micrographs of rat kidney treated with Etoposide for 8 weeks. Note the few number of mitochondria (M) with electron dense matrix. Nucleus ( $\mathrm{N}$ ) with chromatin condensation. Few lysosomes (Ly) are also seen. Also note the smooth endoplasmic reticulum (SER) with amorphous material. Magnification taken (x 10,000).

indicative of marked congestion of the glomeruli with glomerular atrophy. Branden et al. [16] found similar results in case of adriamycin treated rats and Kim et al. [17] in case of cisplatin treated rabbit.

However adriamycin, which is also an topoisomerase-II inhibitor showed chronic renal failure with the development of both interstial anf glomerular sclerosis. Tubulo-interstial lesion were also apparent after adriamycin treatment [16].
Current investigations by using light microscopy depicts vacuolations in the epithelial lining of proximal convoluted tubule (PCT), whereas desquamation in particular was not noticed, but damage to the brush border of the cell and presence of debris in the tubular lumen was apparent. Almost similar changes were found in adriamycin-treated renal tissue where collagen in glomerulus and in the tubulo-interstial compartment was observed, found to be significantly increased in rats [16]. Ultrastructural studies in etoposide treated renal tissue have not been documented so far. Present observations on etoposide treated renal tissue after electron microscopy presented several ultrastructural changes in S1, S2 and S3 segments of the proximal tubules. The $\mathrm{S} 2$ segment showed presence of scanty microvilli of variable height; whereas S3 segment showed complete abscence of microvilli. Since the brush border membrane plays important role in proximal tubular reabsorption processes [17], an impaired aminoacid and glucose reabsorption can result from its alterations. This has also been reported previously in rats with ischemic or Cd-induced renal failure $[17,18]$. Absence of microvilli in S3 segment of proximal convoluted tubule (PCT) as in the present study is in good correlation with findings of these workers. This clearly indicates that etoposide also has similar effects on proximal convoluted tubule (PCT) in glucose and amino acid reabsorption like that of cadmium toxicity.

The presence of many mitochondria of variable lengths with dense matrix along basolateral folding was indicative of respiratory impairment [19]. Basal energy consumption rate of renal tissue is usually high under stress condition inorder to compensate the higher requirement of energy; the renal tissue undergoes the changes in its subcellular distribution and shape of mitochondria. However, study demands the need to access the polymorphic nature of mitochondria within the cells [20]. Renal tissue of etoposide treated rats showed increased population of polymorphic mitochondria with dense matrix in our investigation. In correlation with the overwhelming statement made by Lash et al. [20] it can be thus said that etoposide may have caused stress in the renal tissue. To overcome this stress response the mitochondria population and polymorphism was increased.

Renal tissue of etoposide treated rats also showed condensation of nuclear chromatin and appearance of lysosomal bodies. The presence of several lysosomal bodies in any cell might be indicating the degenerative activity. Biochemical findings of Gpx showed significant increase in its activity after etoposide treatment in our studies. Thus, presence of lysosomes in the tissue and corrseponding increase in Gpx activity perhaps is a indication of degenerative activity in the renal tissue of treated rats. Role of smooth endoplasmic reticulum (SER) in detoxification and drug metabolism is a known fact [21]. An active process of detoxification in the renal tissue after etoposide treatment in present study was clear, due to the presence of SER with amorphous material.

The presence of necrotic debris in the tubular lumen with tubular damage was an indication of onset of necrosis. The histopathological and ultrastructural changes in the renal tissue after etoposide treatment are supported by biochemical evaluations of various antioxidant enzymes. Our results demonstrated that etoposide can act as an antioxidant which is indicated by decreases Lpx levels in kidney. It is possible that it can act as a potential antioxidant against $\mathrm{H}_{2} \mathrm{O}_{2}$ induced phospholipid peroxidation.

Kagan et al. [3] have reported for the first time that etoposide does not cause peroxidation of any of major phospholipid in HL-60 cells. Lipid peroxidation in our studies also showed non-significant decrease 
in the treated tissue. Thus it can be conveniently said that at given dose level and duration etoposide does not cause Lpx in renal tissue in vivo.

Katki et al. [22] have showed that VP-16 increase total thiol pools in kidney is due to significant increase in GSH or thiol status. In our studies the GSH levels after etoposide treatment is not in accordance with this report. Our results showed an insignificant decrease from control values, probably indicates least requirement of thiols for removal of VP-16 free radical. All these above changes in the ultrastructural studies and biochemical parameters discussed so far.

Significantly increased activity of G-S-T in kidney after etoposide treatment probably indicates that the enzyme acts as a marker for any oxidative stress by the drug [23]. However, the above given reference is pertaining to adriamycin effects on tumor cell line is avaliable. As far as etoposide is concerned there are no reports made in clinical oncology on long- term effects of etoposide on the kidney.

GR activities have shown significant decrease, which might have occured due to decreased oxidized GSH in the tissue. On the contrary Katki et al. [22] have demonstrated that administration of VP-16 to mice increase the total pools in kidney with a significant increase GR activity. Various other investigators have previously reported that $\mathrm{AOE}$ and ROI contents may change considerably in diseased organs and kidney [16]. In this study, we have reported that after 60 days, etoposide toxicity is manifested in the form of significant increase in renal Gpx activity and non-significant decrease in CAT activity. This supports the idea that CAT and Gpx in the tissue is more vulnerable to oxidative stress in renal diseases [24] but an non-significant change in CAT perhaps is an indication, that increased Gpx is capable of compensating for the reduced activity of CAT inorder to increase the tolerance of the tissue to oxidative stress.

The relevance of Cyp450 has long been implicated in the metabolism of anticancer drugs such as cyclophosphoamide, procarbazine and etoposide [25], also multiple dosage of anticancer drugs is responsible for increase in Cyp450. Lowered levels of Cyp450 perhaps have resulted into lowered drug metabolism which may have further resulted in decreased levels of Cyp b5. The studies also suggests that lower Cyp450 activation might also have least free radical formation which is evident of lower lipid peroxidation and lesser enzyme inactivation as seen in our studies.

In the current studies GSH shows insignificant decrease in the treated samples. Thus significant decrease in GGT activity can be accounted for non-significant reduction in GSH levels.

However, lower activity of GGT indicates decrease in the metabolic activation of quinines or any drug and even etoposide. This also indicates lower ability of the drug to damage the renal tissue at given dose levels and duration.

To sum up it can be said that etoposide at given dose level and duration may cause minor structural and biochemical changes. Our results at histological, fine structural and biochemical levels are in good accordance with each other.

\section{Acknowledgement}

We would express our sincere thanks to all members of the lab. Our special thanks to all team of Jaslok hospital and Research Center Dr. Arun Chitale, Mr. Kanaskar, Mr. Shivaji Bhosale and Mr. Mahadik for their assistance with the electron microscopic work.

\section{References}

1. Imbert TF (1998) Discovery of podophyllotoxins. Biochimie 80: 207-222.
2. Henwood JM, Brogden RN (1990) Etoposide. A review of its pharmacodynamic and pharmacokinetic properties, and therapeutic potential in combination chemotherapy of cancer. Drugs 39: 438-490.

3. Kagan VE, Yalowich JC, Borisenko GG, Tyurina YY, Tyurin VA, et al. (1999) Mechanism-based chemopreventive strategies against etoposide-induced acute myeloid leukemia: free radical/antioxidant approach. Mol Pharmacol 56: 494-506.

4. Ladner C, Ehninger G, Gey KF, Clemens MR (1989) Effect of etoposide (VP16-213) on lipid peroxidation and antioxidant status in a high-dose radiochemotherapy regimen. Cancer Chemother Pharmacol 25: 210-212.

5. Relling MV, Nemec J, Schuetz EG, Schuetz JD, Gonzalez FJ, et al. (1994) O-demethylation of epipodophyllotoxins is catalyzed by human cytochrome P450 3A4. Mol Pharmacol 45: 352-358.

6. RACKER E (1955) Glutathione reductase from bakers' yeast and beef liver. J Biol Chem 217: 855-865.

7. Rotruck JT, Pope AL, Ganther HE, Swanson AB, Hafeman DG, et al. (1973) Selenium: biochemical role as a component of glutathione peroxidase. Science 179: $588-590$

8. Indirani N, Hill PG (1977) Partial purification and some properties of ?-glutamy transpeptidase from human bile. Biochem Biophys Acta $179: 588-590$.

9. Habig WH, Pabst MJ, Jakoby WB (1974) Glutathione S-transferases. The first enzymatic step in mercapturic acid formation. J Biol Chem 249: 7130-7139.

10. Aebi $\mathrm{H}$ (1984) Catalase in vitro. Methods Enzymol 105: 121-126.

11. Moron MS, Depierre JW, Mannervik B (1979) Levels of glutathione, glutathione reductase and glutathione $S$-transferase activities in rat lung and liver. Biochim Biophys Acta 582: 67-78.

12. Esterbauer H, Cheeseman KH (1990) Determination of aldehydic lipid peroxidation products: malonaldehyde and 4-hydroxynonenal. Methods Enzymol 186: 407-421.

13. Omura T, Sato R (1964) The carbon monoxide-binding pigment of liver microsomes. I. Evidence for its hemoprotein nature. J Biol Chem 239: 2370 2378.

14. Lowry OH, Rosebrough NJ, Farr AL, Randall RJ (1951) Protein measurement with the Folin phenol reagent. J Biol Chem 193: 265-275

15. Humason, Gretchen L (1979) Animal tissue techniques, WH Freema and Co. San Franciso.

16. Van den Branden C, Ceyssens B, De Craemer D, Pauwels M, Vanden Houte $\mathrm{K}$, et al. (2000) Renal antioxidant enzymes and fibrosis-related markers in the rat adriamycin model. Nephron 86: 167-175.

17. Kim YK, Byun HS, Kim YH, Woo JS, Lee SH (1995) Effect of cisplatin on renal function in rabbits: mechanism of reduced glucose reabsorption. Toxicol Appl Pharmacol 130: 19-26.

18. Molitoris BA, Kinne R (1987) Ischemia induces surface membrane dysfunction Mechanism of altered $\mathrm{Na}$ +-dependent glucose transport. J Clin Invest 80: 647654.

19. Kharbangar A, Khynriam D, Prasad SB (2000) Effect of cisplatin on mitochondrial protein, glutathione, and succinate dehydrogenase in Dalton lymphoma-bearing mice. Cell Biol Toxicol 16: 363-373.

20. Lash LH, Visarius TM, Sall JM, Qian W, Tokarz JJ (1998) Cellular and subcellular heterogeneity of glutathione metabolism and transport in rat kidney cells. Toxicology 130: 1-15.

21. Farber $E$ (1984) Cellular biochemistry of the stepwise development of cance with chemicals: G. H. A. Clowes memorial lecture. Cancer Res 44: 5463-5474.

22. Katki AG, Kalyanaraman B, Sinha BK (1987) Interactions of the antitumor drug etoposide, with reduced thiols in vitro and in vivo. Chem Biol Interact 62: $237-$ 247.

23. Habig WH, Pabst MJ, Jakoby WB (1974) Glutathione S-transferases. The first enzymatic step in mercapturic acid formation. J Biol Chem 249: 7130-7139.

24. Tsukamoto M, Tampo Y, Sawada M, Yonaha M (2002) Paraquat-induced oxidative stress and dysfunction of the glutathione redox cycle in pulmonary microvascular endothelial cells. Toxicol Appl Pharmacol 178: 82-92.

25. Nebert DW, Russell DW (2002) Clinical importance of the cytochromes P450. Lancet 360: 1155-1162. 